\title{
Approximately Optimal Wireless Broadcasting
}

\author{
Sreeram Kannan, Adnan Raja, and Pramod Viswanath, Senior Member, IEEE
}

\begin{abstract}
We study a wireless broadcast network, where a single source reliably communicates independent messages to multiple destinations, with the potential aid of relays and cooperation between destinations. The wireless nature of the medium is captured by the broadcast nature of transmissions as well as the superposition of transmitted signals plus independent Gaussian noise at the received signal at any radio. We propose a scheme that can achieve rate tuples within a constant gap away from the cut-set bound, where the constant is independent of channel coefficients and power constraints. First, for a deterministic broadcast network, we propose a new coding scheme, constructed by adopting a "receiver-centric" viewpoint, that uses quantize-and-forward relaying as an inner code concatenated with an outer Marton code for the induced deterministic broadcast channel. This scheme is shown to achieve the cut-set bound evaluated with product form distributions. This result is then lifted to the Gaussian network by using a deterministic network called the discrete superposition network as a formal quantization interface. This two-stage construction circumvents the difficulty involved in working with a vector nonlinear non-Gaussian broadcast channel that arises if we construct a similar scheme directly for the Gaussian network.
\end{abstract}

Index Terms-Broadcast network, broadcast-relay channels, capacity, Marton code, multiuser channels, network information theory, wireless networks.

\section{INTRODUCTION}

$\mathbf{T}$ HE scenario of study in this paper is a communication network with broadcast traffic, as illustrated in Fig. 1. Broadcast here means that a single source node is reliably communicating independent messages to multiple destination nodes using the help of multiple relay nodes. In the example of a cellular system, the setting represents down-link communication where the base-station is transmitting to multiple terminals with the potential help of relay stations. Note that some of the terminals can themselves act as relays.

The term wireless most commonly refers to the Gaussian network model, where the canonical Gaussian channel model describes the relationship between the transmitted and received symbols of the various nodes in the network. We assume that time is assumed to be discrete and synchronized among all

Manuscript received November 26, 2010; revised January 04, 2012; accepted March 20, 2012. Date of publication August 03, 2012; date of current version November 14, 2012. This work was supported in part by the National Science Foundation under Grants CCF 1017430 and CNS 0721652. The material in this paper was presented in part at the International Conference on Signal Processing and Communications, Bangalore, India, 2010, and in part at the 2011 IEEE International Symposium on Information Theory.

S. Kannan and P. Viswanath are with the University of Illinois, Urbana-Champaign, Champaign, IL 61801 USA (e-mail: kannan1@illinois.edu; pramodv@illinois.edu).

A. Raja was with the University of Illinois, Urbana-Champaign, Champaign, IL 61801 USA. He is now with Stanford University, Stanford, CA 94305 USA (e-mail: araja2@illinois.edu).

Communicated by R. Yates, Associate Editor for Communication Networks.

Color versions of one or more of the figures in this paper are available online at http://ieeexplore.ieee.org.

Digital Object Identifier 10.1109/TIT.2012.2211566
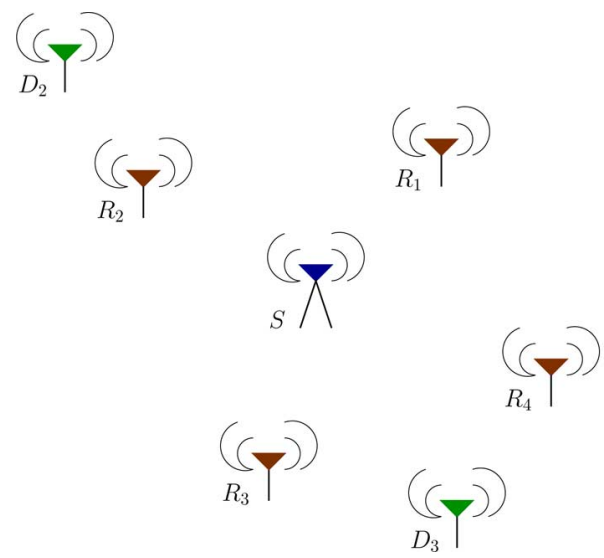

Fig. 1. Wireless broadcast network.

the nodes. Denoting the baseband transmit symbol (a complex number) of node $k$ at time $t$ by $x_{k}[t]$, the average transmit power constraint at each node implies that

$$
\sum_{t=1}^{T}\left|x_{k}[t]\right|^{2} \leq T P_{k}
$$

where $T$ is the time period over which the communication occurs. At each time $t$, we have the received signal at any node $\ell$

$$
y_{\ell}[t]=\sum_{k \neq \ell} h_{k \ell}[t] x_{k}[t]+z_{\ell}[t]
$$

Here, $\left\{z_{\ell}[t]\right\}_{t}$ is i.i.d. Gaussian noise and independent across the different nodes $\ell$. The channel attenuation $h_{k \ell}$ between a pair of nodes $(k, \ell)$ is supposed to be constant over the time scale of communication. Note that by normalizing the channel attenuation $h_{k \ell}$, without loss of generality, we will assume unit average power constraints at each node, i.e., $P_{k}=1$ and also the variance of $z_{\ell}[t]$ to be 1 . We suppose full duplex mode of operation for the most part, except while discussing the implications of half-duplex mode later in the paper. We suppose single antenna at each node throughout the paper except in an explicitly mentioned section where the ramifications of the presence of multiantenna nodes are explored. We will begin with the supposition that these channel attenuations are known to all the nodes in the network, and revisit this requirement later.

In [1], a deterministic approach was introduced to study wireless networks. A linear-deterministic network was introduced, where the inputs and outputs are vectors over a finite field, i.e., $\mathcal{X}_{j}=\mathcal{Y}_{j}=\mathbb{F}_{p}^{q}$, for some prime $p$ and $q \in \mathbb{N}$. The channels are linear transformations over this finite field, i.e.,

$$
y_{j}[t]=\sum_{i \in N_{j}} G_{i, j} x_{i}[t]
$$

where $G_{i, j} \in \mathbb{F}_{p}^{q \times q}$. In particular, $G_{i, j}$ are often assumed to be "shift" matrices, which capture channel attenuation of the wire- 
less link. The linear deterministic model, on the one hand, captures wireless signal interactions like interference and broadcast and, on the other hand, has an algebraic structure that can be exploited for understanding schemes in this network.

The linear-deterministic network, however, has shortcomings; in particular, it does not capture the phase of the channel attenuations and, therefore, does not approximate the capacity of the Gaussian network. To overcome these shortcomings, a deterministic network called the discrete superposition network (DSN) was introduced in [2] and it was shown that the DSN can act as a digital interface to construct schemes for the Gaussian relay network. Our approach in this paper is to extend this construction to the Gaussian broadcast network. We construct and characterize the coding scheme for any deterministic broadcast network - the DSN being a special case. We then use the DSN as a digital interface to construct a scheme for the Gaussian network. The DSN will be described in detail in Section IV-A.

The broadcasting setup we present here captures two important special cases, which have been extensively studied before: the unicast wireless relay network and the broadcast channel $(B C)$.

An approximately optimal scheme for the unicast wireless relay network was given recently in [1]. The scheme consists of each relay node doing a quantize-map-forward operation. The existence of a good mapping was shown by considering a random ensemble of mappings and averaging performance across this. It was shown that this scheme was approximately optimal in the sense that it achieves rates, within a constant gap of the well-known cut-set outer bound, where the constant gap does not depend on the power and the channel parameters but only on the size of the network. The scheme was based on insight derived from the scheme in the deterministic network in the same paper. Lim et al. [5] give a general coding scheme, called the noisy network coding scheme, for the discrete-time memoryless relay network. Specialized to the Gaussian network, it gives similar approximation results as [1]. In [2], yet another alternate approach was provided, wherein the DSN was used as a digital interface for the Gaussian network and the scheme was constructed by lifting the scheme for the DSN.

The $\mathrm{BC}$ is a special case with only a source node and multiple destinations (i.e., no relays). The capacity of the Gaussian BC with multiple antennas (MIMO BC) was characterized in [11]. The capacity achieving scheme is based on the Marton's coding scheme [6], which is the best known achievable scheme for the general $\mathrm{BC}$.

The coding scheme presented here for broadcasting in wireless relay networks is loosely based on combining the quantize-map-and-forward scheme for the relay network and Marton's coding scheme for the induced nonlinear non-Gaussian BC. Broadcasting in wireless networks has been studied previously in a special scenario called the broadcast relay channel in [8], which comprises of a two-user $\mathrm{BC}$ where the destination nodes could also transmit, thereby also acting as relay nodes. Decode-and-forward schemes were considered and specialized outer bounds were given for this network, which were shown to be better than cut-set bounds. More recently, Vasudevan and Korada [26] have considered broadcasting over two classes of information networks-1) a network composed of multiple-access channels (MACs) alone and 2) a network composed of deterministic BCs alone. For such networks, it was shown there that cut-set bound can be achieved. The scheme proposed there is a separation based scheme-a local physical layered scheme over the constituent networks to create a wired overlay network and a global routing scheme over the overlay network.

In contrast, in this paper, we deal with general deterministic broadcast networks and Gaussian broadcast networks, for both of which we show approximate capacity results using a nonseparation-based scheme.

\section{A. Lesson From Reciprocity}

The key intuition motivating the scheme presented here is the lesson learnt from reciprocity. Note that the reciprocal of the broadcast network is the network with multiple sources and a single destination. It was shown in [5] and [17] that schemes for the unicast network naturally extend to the multi-source single-destination case. These schemes were further shown to be approximately optimal by comparing them to the cut-set bound. Reciprocity would suggest the existence of similar schemes for the broadcast network.

In going from the multi-source to the reciprocal broadcast case, certain difficulties naturally arise. These difficulties were seen even in the simple case of the multiple-access and the BC. While the capacity schemes for the MAC generalized simply from the point-to-point case, schemes for the BC involved clever coding at the source node. The difference can be attributed to where (at the transmitter or the receiver) the complexity of the scheme lies. For the MAC, the complexity lies at the decoder. It is easy to show the existence of good coding schemes for the MAC using simple random codebooks and analyzing performance of the complex joint decoder. For the BC (reciprocally), the complexity is at the encoder. The complex encoding is often elusive. In fact, for a general BC, the optimal scheme and the capacity are still unknown. The best known scheme is a family of schemes due to Marton [6] which is optimal only for certain special cases. For the Gaussian channel with multiple antennas it is known that a particular Marton coding scheme (called Costa's dirty paper coding) is optimal [11], [22], [23]. For the degraded BC, superposition coding is optimal. For the deterministic channel, a simple Marton coding scheme achieves the cut-set bound [9].

Carrying this intuition forward to networks, the scheme for the multiple-source network suggests the following scheme for the broadcast network. The relays and the destination node perform a quantize-map-forward operation. The source takes into account the effect of the channel and operations performed by the relay node and needs to do a well-designed scheme for the effective $\mathrm{BC}$. The limitations on the understanding of the $\mathrm{BC}$ restrict us to design such a scheme only when the channel model is deterministic. Even when the channel model is linear with Gaussian noise, taking into account the relay operation leads to an end-to-end nonlinear non-Gaussian vector BC.

The main technical contribution is the leveraging of the DSN as a formal quantization interface for the Gaussian network to circumvent this difficulty and to prove coding theorems for the Gaussian network. Coding schemes for the DSN are simpler to 
derive because of the deterministic nature of the network. The rest of this paper makes these notions precise. After discussing the approximately optimal scheme for the broadcast network, we will revisit the notion of reciprocity in Section II-B.

\section{B. Communication Setup}

Let $\mathcal{V}$ denote the set of all the nodes with $|V|=n$. Let $X_{v} \in$ $\mathcal{X}_{v}, Y_{v} \in \mathcal{Y}_{v}$ specify the transmit receive symbols at node $v$ drawn from the alphabet $\mathcal{X}$ and $\mathcal{Y}$, respectively. A memoryless network is specified by the following transition probability:

$$
p\left(Y_{1}, \ldots, Y_{n} \mid X_{1}, \ldots, X_{n}\right)
$$

A broadcast network is a memoryless network specified by the a distinguished node called the source $S$ and $J$ destinations $D_{1}, D_{2}, \ldots, D_{J}$. The source wants to deliver independent information to each of the $J$ destinations at rates $\left(R_{1}, \ldots, R_{J}\right)$.

A $\left(2^{T R_{1}}, \ldots, 2^{T R_{J}}, T\right)$ coding scheme for the broadcast network over $T$ time instants is comprised of the following.

1) Independent random variables $W_{i}$ which are distributed uniformly on $\left\{1, \ldots, 2^{T R_{i}}\right\}$ for $i=1, \ldots, J$, respectively. $W_{i}$ denotes the message intended for destination $D_{i}$.

2) The source mapping for time $t$

$$
f_{S, t}:\left(W_{1} \times \cdots \times W_{J}, \mathcal{Y}_{S}^{t-1}\right) \rightarrow \mathcal{X}_{S}^{t} .
$$

3) The relay mappings for each $v \in \mathcal{V} \backslash\{S\}$ and time $t$

$$
f_{v, t}: \mathcal{Y}_{v}^{t-1} \rightarrow \mathcal{X}_{v}
$$

4) The decoding map at destination $D_{i}$

$$
g_{D_{i}}: \mathcal{Y}_{D_{i}}^{T} \rightarrow \hat{W}_{i}
$$

The probability of error for destination $D_{i}$ under this coding scheme is given by

$$
P_{e}^{i} \stackrel{\text { def }}{=} \operatorname{Pr}\left\{\hat{W}_{i} \neq W_{i}\right\} .
$$

A rate tuple $\left(R_{1}, R_{2}, \ldots, R_{J}\right)$, where $R_{i}$ is the rate of communication in bits per unit time for destination $D_{i}$, is said to be achievable, if for any $\epsilon>0$, there exists a $\left(2^{T R_{1}}, 2^{T R_{2}}, \ldots, 2^{T R_{J}}, T\right)$ scheme that achieves a probability of error lesser than $\epsilon$ for all nodes, i.e., $\max _{i} P_{e}^{i} \leq \epsilon$. The capacity region $\mathcal{C}$ is the set of all achievable rates.

\section{Cut-Set Outer Bound}

The following is the well-known cut-set outer bound to the rate tuples of reliable communication [3], [4]:for a subset $\mathcal{J} \subseteq$ $[J]$, where $[J]$ denotes the set $\{1, \ldots, J\}$, denote $\Lambda_{\mathcal{J}}$ to be the collection of all subsets $\Omega \subset \mathcal{V}$, such that the source node $S \in \Omega$ and the subset of destination nodes $D_{\mathcal{J}} \stackrel{\text { def }}{=}\left\{D_{i} \mid i \in \mathcal{J}\right\} \subseteq \Omega^{c}$. If $\left(R_{1}, \ldots, R_{J}\right)$ is achievable, then there is a joint distribution $p\left(\left\{X_{v} \mid v \in \mathcal{V}\right\}\right)$ (denoted by Q) such that

$$
R_{\mathcal{J}} \leq \bar{C}_{\mathcal{J}}(Q) \stackrel{\text { def }}{=} \min _{\Omega \in \Lambda_{\mathcal{J}}} I\left(Y_{\Omega^{c}} ; X_{\Omega} \mid X_{\Omega^{c}}\right) \quad \forall J \subseteq[J]
$$

where $R_{\mathcal{J}} \stackrel{\text { def }}{=} \sum_{j \in \mathcal{J}} R_{j}$.
Let $\overline{\mathcal{C}}(Q)$ denote the set of all rate tuples that satisfy the cut-set outer bound for a given joint distribution $Q$, and $\overline{\mathcal{C}}$ denote the cut-set bound:

$$
\begin{aligned}
& \overline{\mathcal{C}}(Q) \stackrel{\text { def }}{=}\left\{\left(R_{1}, \ldots, R_{J}\right): R_{\mathcal{J}} \leq \bar{C}_{\mathcal{J}}(Q) \quad \forall \mathcal{J} \subseteq[J]\right\} \\
& \overline{\mathcal{C}} \stackrel{\text { def }}{=} \bigcup_{\left\{Q: \mathbb{E}\left|X_{v}\right|^{2} \leq 1\right\}} \overline{\mathcal{C}}(Q) .
\end{aligned}
$$

The capacity region is contained inside the cut-set bound, i.e., $\mathcal{C} \subseteq \overline{\mathcal{C}}$

The cut-set bound under product-form distributions is given by

$$
\overline{\mathcal{C}}_{p} \stackrel{\text { def }}{=} \bigcup_{\left\{Q_{p} \in \mathcal{Q}_{p}: \mathbb{E}\left|X_{v}\right|^{2} \leq 1\right\}} \overline{\mathcal{C}}\left(Q_{p}\right)
$$

where $\mathcal{Q}_{p}$ is the set of all distributions $p\left(\left\{X_{v} \mid v \in \mathcal{V}\right\}\right)$ which have a product form $\prod_{\{v \in \mathcal{V}\}} p\left(X_{v}\right)$. Clearly $\overline{\mathcal{C}}_{p} \subseteq \overline{\mathcal{C}}$, since the latter is a larger union.

\section{Main Result}

Our main result is the following.

Theorem 1: For the Gaussian broadcast network, given a tuple $\left(r_{1}, \ldots, r_{J}\right) \in \overline{\mathcal{C}}$ that lies in the cut-set region, any rate tuple $\left(R_{1}, \ldots, R_{J}\right)$ satisfying

$$
\sum_{j \in \mathcal{J}} R_{j} \leq \sum_{j \in \mathcal{J}} r_{j}-k \quad \forall \mathcal{J} \subseteq(1,2, \ldots, J)
$$

is achievable where $k=O(|\mathcal{V}| \log |\mathcal{V}|)$ is a constant which depends only on the number of nodes, and not on the channel coefficients or the power constraints.

Corollary 1: If $\left(r_{1}, \ldots, r_{J}\right) \in \overline{\mathcal{C}}$, a rate vector $\left(r_{1}\right.$ $\left.k, \ldots, r_{J}-k\right)$ is achievable where $k=O(|\mathcal{V}| \log |\mathcal{V}|)$.

The approach to prove the above theorem is the following. A coding theorem is first proved for the deterministic network. The proposed scheme operates in two steps. The inner code, in which the relays essentially perform a quantize-map-and-forward operation. This induces a vector BC end-to-end between the source node and the destination nodes. The outer code is a Marton code (see [6] and [7]) for the BC induced by the relaying scheme.

The coding scheme for the DSN is then lifted to the Gaussian network, by using the DSN as a digital interface for the Gaussian network.

The rest of the paper is organized as follows. In Section II, we discuss linear-deterministic networks, reciprocity and some challenges in designing schemes for Gaussian networks. This section provides the intuition based on which schemes are designed in later sections. In Section III, we give a coding scheme and establish an achievable rate region for (general) deterministic broadcast networks. In Section IV, we prove Theorem 1 by giving a coding scheme for the Gaussian network. In Section V, various generalizations of the scheme are provided, for halfduplex networks, for networks with multiple antenna and for broadcast wireless networks, where some set of nodes demand independent information and other nodes demand all the information. In Section VI, we discuss various aspects of the pro- 
posed scheme, primarily reciprocity in the context of Gaussian networks, and the channel state information (CSI) required.

\section{LINEAR-DETERMINISTIC NETWORKS AND RECIPROCITY}

In this section, we first discuss the special case of linear-deterministic broadcast networks, and capacity-achieving schemes for such networks. We then revisit the notion of reciprocity with a particular emphasis on the contrast between transmitter-centric and receiver-centric viewpoints to the schemes. Next, we discuss an example which highlights the challenges in designing schemes for the broadcast relay network. This example shows that coordination needs to be induced at the relays and how a receiver-centric scheme can perform such coordination.

\section{A. Linear-Deterministic Networks}

A deterministic network of particular interest is the linear finite-field broadcast deterministic network [1]. The inputs and outputs are vectors over a finite field, i.e., $\mathcal{X}_{j}=\mathcal{Y}_{j}=\mathbb{F}_{p}^{q}$, for some prime $p$ and $q \in \mathbb{N}$. The channels are linear transformations over this finite field, i.e.,

$$
y_{j}[t]=\sum_{i \in N_{j}} G_{i, j} x_{i}[t]
$$

where $G_{i, j} \in \mathbb{F}_{p}^{q \times q}$.

An important question is whether simple linear schemes are optimal for these networks. It has already been shown in [1] that for the single-source single-destination relay network, linear mappings at all nodes suffice. The intuition behind the proof is that, when the relay nodes randomly pick transformation matrices, the resulting matrix between the source and the destination has rank equal to the min-cut rank of the network, with high probability. Therefore, if the rate is lesser than the min-cut rank, random linear coding at all nodes (including the source but not the destination) ensures an end-to-end full-rank matrix and the destination, knowing all these encoding matrices, picks up a decoding matrix, which is the inverse of the end-to-end matrix. This intuition is then used to obtain schemes in the general deterministic relay network and the Gaussian relay network in [1], where the relays perform random mapping operations resulting in an induced end-to-end channel between the source and the destination. Then, the source uses a random code to map the messages, and the destination performs a typical set decoding. It has also been shown in [12] and [13] that for the linear-deterministic relay network, restricting the relay mappings to permutation matrices is without loss of optimality. The next corollary claims a similar result even for the linear-deterministic broadcast network.

Corollary 2: For linear-deterministic broadcast network, linear coding at every node is sufficient to achieve capacity. Furthermore, the mapping at relay nodes can be restricted to permutation matrices.

Although this can be proved directly, and is already shown in [19] using an algebraic approach, we will use the connection between linear coding and reciprocity to prove this in the next section as this will form the intuitive basis for our schemes in more general settings.

\section{B. Reciprocity}

In Section I-A, we pointed out that our scheme was inspired from the consideration of reciprocity in networks. We revisit the notion here again, now with the hindsight of the scheme to better understand our intuitions.

Generally speaking, by the reciprocal of a network, we mean a network where the direction of links are reversed and the roles of the source and destination are swapped. For a Gaussian network, with power constraints at the transmitting nodes and additive noise at the receiver nodes, it is not clear what is the most appropriate way of defining the corresponding reciprocal network. However, we will use the canonical definition of the reciprocal of a Gaussian network having unit power constraint at all nodes and unit noise variance with multiple unicast traffic as the network where the roles of the sources and the destinations are swapped, and all nodes retain their unit power constraint and unit noise variance at the receivers. Note that any channel coefficient that captures the signal attenuation between a pair of nodes is the same in either direction. For a linear-deterministic network, the reciprocal network was defined in [14] as the network where the roles of the sources and the destinations are swapped, and the channel matrices are chosen as transposes of each other in the forward channel for the network and its reciprocal.

While it is unresolved whether a given network and its reciprocal have the same capacity region [27], many interesting examples are known for which this is true. For some cases, this reciprocity is applicable even at the scheme level.

1) Wireline networks can be considered as a special case of wireless networks studied here. It has been shown in [21] that wireline networks are reciprocal (also called reversible in the literature) under linear coding.

2) In [14], it was shown that reciprocity, under linear coding, can be extended naturally to the linear-deterministic network. The reciprocity was shown at the scheme level and the coding matrices at each node can be obtained from the coding matrices in the reciprocal network.

3) In Gaussian networks, duality has been shown (see [22] and [23]) between the MAC and BC, where it was shown that the capacity region of the MAC is equal to the capacity region of the $\mathrm{BC}$ under the same sum power constraint. This duality was also shown, interestingly, at the scheme level between the dirty-paper precoding for the $\mathrm{BC}$ and the successive cancellation for the MAC.

The reciprocal network corresponding to the broadcast network studied here is the network with many sources and one destination. This network has been studied in [5], [17], and [24].

1) Sufficiency of Linear Coding for Linear-Deterministic Networks: In [24], the capacity region for the linear-deterministic network with many sources and one destination is established and it is further shown that linear coding is sufficient to achieve this. This is done by converting the problem to the case of single-source single-destination by adding a super-node and connecting all the source nodes to the super-node by orthogonal links with capacities equal to the rate required for that source. Since random linear coding at the source and the relays works for the single-source single-destination 
network, it works for this network too. Therefore, the source nodes and the relay nodes perform random mappings, and the destination, knowing the source and relay mappings, can then carefully pick the decoding matrix that inverts this overall matrix. Since this coding is linear, we can use the reciprocity result of [14], to show that any rate achievable in the dual multiple-source single-destination network is also achievable in the single-source multiple-destination case. Along with the fact that the cuts are reciprocal in these two networks, this implies that linear coding is optimal even in the case of the linear-deterministic broadcast network.

Furthermore, from the results of [12] and [13], it can also be shown that the sources and the relays can pick up specific permutation matrices for the single-source single-destination network. The above argument can then the extended to show that a coding scheme involving only permutation mappings at the relays is sufficient for the linear-deterministic broadcast network.

2) Receiver-Centric Versus Transmitter-Centric Schemes: We now continue on our discussion on duality for linear-deterministic network to illustrate how these ideas lead us to a scheme for the Gaussian broadcast network. We begin by defining two viewpoints in which schemes can be constructed. A transmitter-centric scheme is one in which the scheme is constructed from the viewpoint of the transmitter, where the codebook at the transmitter is first selected using a random coding argument and then the receiver chooses its decoders in accordance with the realization of the transmit codebook. In contrast, in a receiver-centric scheme, we fix the decoder, which comprises of the mappings from the received vectors to the messages, and based on these mappings, the transmitter chooses its codebook to ensure low probability of error.

Because random coding is done at the source, we can think of this scheme as first constructing the transmitter codebook in a random manner and the receiver then constructs its decoder as a function of the realization of the transmit codebook. While the scheme for the linear-deterministic multi-source network is transmitter-centric, the scheme for the linear-deterministic broadcast network is receiver-centric. We will now study the schemes in several well-known examples and categorize them as transmitter-centric or receiver-centric.

a) Point-to-point channel: For a point-to-point channel, the usual random coding scheme [3] can be regarded as either a transmitter-centric scheme, which is the traditional viewpoint (since the random codebook is thought of as being constructed at the source), or as a receiver-centric scheme. It can be viewed as a receiver-centric scheme, because, at the receiver we construct a vector quantization codebook (alternately viewed as the decoder) of rate $R$, which "quantizes" the received signal $y^{T}$ to a vector $x^{T}(m)$, for some $m$ where $m$ is the message index and $x^{T}(m)$ is the $m$ th quantization codeword. Now the source sets its codebook to be equal to the vector quantization codebook at the destination. This is the same scheme as the usual random coding scheme. The distinction between transmitter-centric and receiver-centric schemes in this example is, therefore, one of personal preference, rather than an enforced one.

b) $M A C$ versus $B C$ : In some networks, we may not have the luxury to use the two viewpoints simultaneously, in which case we need to choose between the two. In the ca- pacity-achieving coding scheme for the MAC [3], the random coding is done at the transmitters and the receiver does joint typical-set decoding, based on the specific codebooks constructed at the sources. This provides a good example of a transmitter-centric scheme.

In contrast, for the two-user $\mathrm{BC}$, we can now view the Marton coding scheme (see [6] and [7]), used in Section III-B3, as a receiver-centric scheme. In this scheme, there are two auxiliary random variables, $U_{1}$ and $U_{2}$, which we view as corresponding to the vector quantization variables at the two users. The receiver $i$ can be thought of as constructing a vector quantization codebook which "quantizes" the received vector $Y_{i}^{T}$ to $U_{i}^{T}\left(w_{i}\right)$, where $w_{i}$ is an index belonging to a set larger than the set of all messages to user $i$, and bins the set of all $w_{i}$ to the message $m_{i}$ for user $i$. The transmitter, to transmit a message pair $\left(m_{1}, m_{2}\right)$, finds a pair $\left(w_{1}, w_{2}\right)$ such that $U_{1}^{T}\left(w_{1}\right), U_{2}^{T}\left(w_{2}\right)$ is jointly typical. From this viewpoint, the receivers are choosing random decoders, and the transmitters are choosing specific codebooks to be a function of the realization of the decoders. Thus, the coding scheme can be viewed as a receiver-centric one.

c) Multiple access versus broadcast in linear-deterministic networks: From [5] and [17], we know that a transmitter-centric scheme, where the sources and the intermediate nodes perform random coding, is optimal for the many-source single-destination problem in the linear determinstic setup. Intuition suggests that a natural receiver-centric method should work for the reciprocal network (i.e., single-source multiple-destination network). In particular, the relays perform random mappings, and the destinations perform "random decoding," i.e., they fix a random linear mapping from the received vector into a smaller message vector. Once these mappings at the relay and the destinations are fixed, the source evaluates the induced linear BC between the source and the various destinations; and constructs a linear broadcast code for this channel. This scheme can then be shown to be optimal for the broadcast network, because this is the reciprocal of the linear random coding scheme, which is optimal for the multi-source single-destination network, as shown in Fig. 2.

d) Scheme for Gaussian broadcast networks as a receivercentric scheme: The general idea for the scheme for the lineardeterministic broadcast network is the foundation of our scheme for the Gaussian broadcast network in Section IV. In order to build the scheme for the Gaussian network, we first construct a scheme for general deterministic networks (of which the DSN is a special case) and then lift the scheme from the DSN to the Gaussian network. In case of the linear-deterministic broadcast network, the source-mapping depended on the specific relay transformations used, not just on the probability distribution used to create the relay transformation. Extending this idea, we would like to construct a scheme for the general deterministic network, where the source codebook is a function of the specific relay transformation. Indeed, we resolve this problem by constructing a Marton scheme at the source for the vector BC induced by the specific relay mappings.

Next, the scheme for lifting codes from the DSN to Gaussian relay networks proposed in [2] requires each node, including the destination, to prune their received vectors to a restricted set to ensure that the received vector in the DSN can be decoded from the received vector in the Gaussian network. Since this scheme 


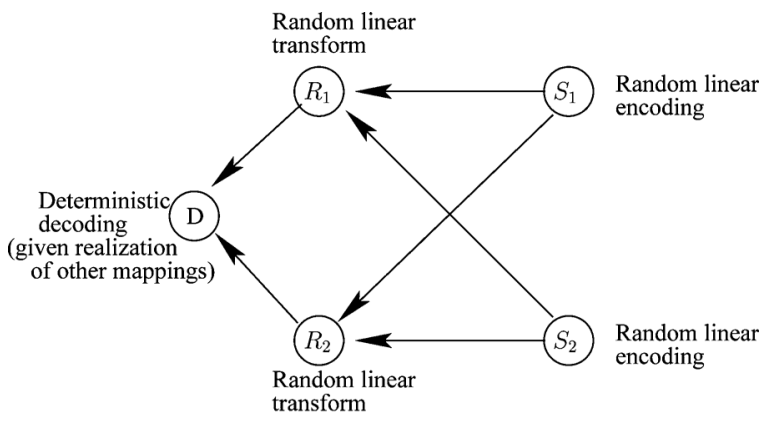

(a)

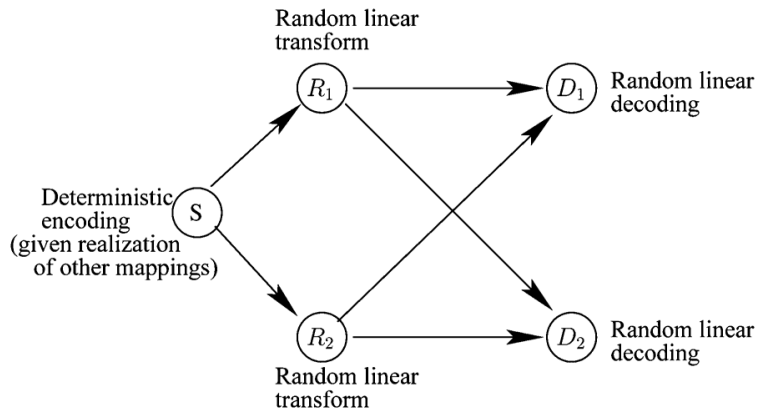

(b)

Fig. 2. Reciprocity in linear-deterministic networks. (a) Random coding scheme for multi-source single-destination network. (b) Dual random coding scheme for single-source multidestination network.

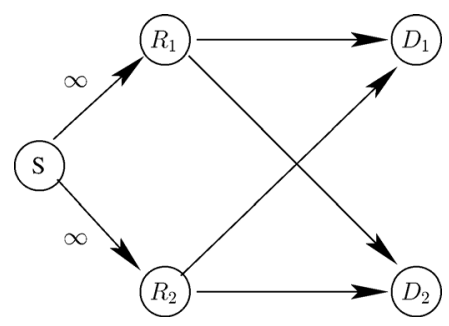

Fig. 3. MISO BC as a special case of broadcast network.

restricts the received codewords at the destination, this scheme also naturally fits into a receiver-centric viewpoint. This enables us to design schemes for Gaussian networks efficiently.

\section{Induced Coordination at Relays}

We now turn to a simple example to illustrate the difficulty in constructing schemes for Gaussian broadcast networks. Let us consider an example Gaussian broadcast network comprised of a single source, two relays, and two destinations, shown in Fig. 3. The link between the source to the two relays is infinite, which implies therefore that the network is essentially an MISO $\mathrm{BC}$ with two transmit antennas and two receivers, each with a single antenna. It is clear [11] that for an MISO BC, independent coding across the two relays is insufficient to even obtain the best possible degrees of freedom. Therefore, any scheme that is approximately optimal needs to perform coordinated transmission at the relays.

In the proposed scheme, the relays perform quantization followed by independent encoding of the quantized bits into transmitted vectors. At a first glance, a scheme in which the relays are performing independent mappings seems incapable of attaining good performance because of the inability to induce coordination. However, two key features in the proposed scheme help avoid this pitfall.

1) The relays $R_{1}$ and $R_{2}$ perform quantize-and-encode relaying in our general scheme, in spite of the fact that they can decode the source message completely. Had the relays decoded the source message and performed independent encoding, there is no possibility of achieving even the degrees of freedom of this simple broadcast network.

2) The source takes into account the specific realizations of the relay mappings and constructs the coding scheme. This ensures that from the point of view of the receiver, the signals transmitted by the two relays appear coordinated. In particular, in this example, since the channel from $S$ to $R_{i}$ is infinitely good, the relay $R_{i}$ quantizes the received signal to a very fine degree and encodes this for transmission to the destination. This gives the source many degrees-offreedom to encode information in the various least significant bits of its transmission, so that after the relay mappings, the relay transmissions appear coordinated.

\section{Deterministic BROADCAST NeTwORKS}

We consider a (general) deterministic or "noise free" network model, where the received signal at each node is a deterministic function of the received signals

$$
y_{\ell}[t]=\psi_{\ell}\left(\left\{x_{k}[t]\right\}_{k \neq \ell}\right) .
$$

The input and output alphabet sets, $\mathcal{X}_{k}$ 's and $\mathcal{Y}_{\ell}$ 's, respectively, are assumed to be finite sets.

While the cut-set outer bound of Section I-C is directly applicable here, we prove the following achievability result for the deterministic channel.

Theorem 2: For the deterministic broadcast network, the region $\overline{\mathcal{C}}_{p}$ is achievable, i.e., a rate vector $\left(R_{1}, \ldots, R_{J}\right)$ is achievable if there is a product distribution $\prod_{v \in \mathcal{V}} p\left(X_{v}\right)$ (denoted by $\left.Q_{p}\right)$ such that

$$
R_{\mathcal{J}} \leq \bar{C}_{\mathcal{J}}\left(Q_{p}\right) \quad \forall \mathcal{J} \subseteq[J]
$$

Remark 1: For many special classes of deterministic networks such as the linear-deterministic network and the network composed of deterministic BCs [26], it can be shown that the cut-set bound is also maximized by the product distribution, thereby characterizing the capacity of such networks completely.

Proof: We prove Theorem 2 for the layered network here. A nonlayered network can be handled by considering a corresponding unfolded network as done in [1]. A network is called an L-layered network if the set of vertices $\mathcal{V}$ can be partitioned into $L$ disjoint sets, such that only the source node $S$ is in the first layer and the $J$ destination nodes are in the $L$ th layer. The nodes in the intermediate layers are relaying nodes. The received signal at the nodes in the $l+1$ th layer only depend on the transmitted signals at the nodes in the $l$ th layer. This dependence is often represented by edges connecting the nodes from the $l$ th layer to the $(l+1)$ th layer. An example of a layered broadcast network is shown in Fig. 4. The advantage of 


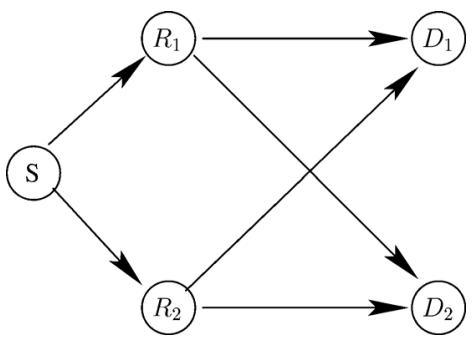

Fig. 4. Layered broadcast-relay network.

working with a layered network is that we can view the information as propagating from one layer to the next without getting intertwined.

\section{A. Outline of Coding Scheme}

The basic idea of the coding scheme is as follows:

1) Let $\left(r_{1}, \ldots, r_{J}\right)$ be a rate tuple in $\bar{C}_{p}$ (the cut-set region of the broadcast network evaluated under product distributions). We will construct a scheme to achieve this rate tuple.

2) The broadcast network is converted into a unicast network by adding a super-destination $D$ which has links from each of the destinations $D_{i}$ by a wired link of capacity $r_{i}$. For this unicast network, the cut-set bound evaluated under product distributions $\bar{C}_{p}^{\text {uc }}$ is equal to $\sum_{j} r_{j}=: r$.

3 ) For the relay network, a zero-error coding scheme is employed that operates over $T_{1}$ time instants, which achieves the rate $r$.

4) The relaying scheme creates an end-to-end deterministic vector $\mathrm{BC}$ between the source and the destinations over vectors of $T_{1}$ symbols.

5) A Marton code is used over a block of $T_{2}$ vectors to achieve the cut-set of the induced deterministic BC, which includes the point $\left(r_{1}, \ldots, r_{J}\right)$.

\section{B. Coding Scheme in Detail}

The random ensemble of coding operations is described for a fixed product distribution $Q_{p}$. Further, the random coding is described to achieve an arbitrary rate tuple $\left(r_{1}, \ldots, r_{J}\right) \in \bar{C}_{p}$. The coding is done over a period of $T_{1} T_{2}$ time instants.

1) Creating a Relay Network: We add a super-sink $D$ to the deterministic broadcast network to obtain a deterministic unicast network. The unicast network is obtained by adding wired links of capacity $r_{i}$ from destination $D_{i}$ to super-sink $D$.

Lemma 1: If for a given $Q_{p},\left(r_{1}, \ldots, r_{J}\right) \in \bar{C}\left(Q_{p}\right)$, then the cut-set $\bar{C}^{\text {uc }}\left(Q_{p}\right)$ of the unicast network with wired links $r_{1}, \ldots, r_{J}$ evaluated under $Q_{p}$ is equal to $r$.

Proof: See Appendix A.

For a deterministic unicast network, Theorem 4.1 in [1] shows that the cut-set under product form distributions is achievable using an $\epsilon$-error scheme. (This is proved by a random coding argument, that shows the existence of a codebook of size $2^{T_{1} r}$ codewords, each codeword of length $T_{1}$ symbols.) Since the channel is deterministic, only an $\epsilon$-fraction of the codewords are decoded with error while the rest are decoded correctly. Discarding these $\epsilon$-fraction of codewords,

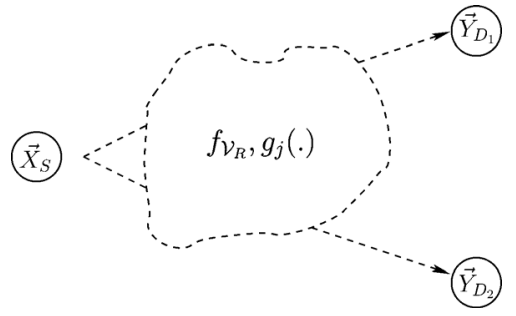

Fig. 5. Effective end-to-end deterministic BC created by an inner code.

leads to negligible rate loss as $T_{1}$ can be chosen arbitrarily large. Therefore, this implies that for the deterministic channel, there is a zero error scheme which can achieve arbitrarily close to the cut-set bound under product form distributions. Thus, the rate $r$ is achievable using such a scheme. Suppose this relaying scheme operates over a block length of $T_{1}$. Let $\vec{x}_{v} \stackrel{\text { def }}{=} x_{v}^{T_{1}}$ and $\vec{y}_{v} \stackrel{\text { def }}{=} y_{v}^{T_{1}}$ denote the transmit and receive block at any node $v \in \mathcal{V}$. Thus, we have a source codebook for the unicast network given by $\mathcal{C}_{S}$, which is a collection of $2^{r T_{1}}$ vectors of length $T_{1}$ each. And the relay mappings

$$
f_{v}: \overrightarrow{\mathcal{Y}}_{v} \rightarrow \overrightarrow{\mathcal{X}}_{v}
$$

for the relay node $v \in \mathcal{V}_{R}$.

2) Relay Mappings: The scheme for the broadcast network operates over $T_{1} T_{2}$ time intervals and this entire time duration is divided into $T_{2}$ blocks, each composed of $T_{1}$ time intervals. Each set of $T_{1}$ time instants is treated as a block and the vector $\vec{x}\left(t_{2}\right)$ denotes $x$ over the $T_{1}$ time instants corresponding to the $t_{2}$ th block: $\vec{x}\left(t_{2}\right)=\left(x\left(\left(t_{2}-1\right) t_{1}+1\right), x\left(\left(t_{2}-1\right) t_{1}+\right.\right.$ $\left.2), \ldots, x\left(t_{2} t_{1}\right)\right)$. Furthermore, $\vec{x}^{T}$ denotes $(\vec{x}(1), \ldots, \vec{x}(T))$.

The relaying operation for the broadcast network is performed in blocks using the relaying scheme for the unicast network as follows. Each relay transmits a $T_{1}$ block using only the information from the previous received $T_{1}$ block. Thus

$$
\vec{x}_{v}\left(t_{2}\right)=f_{v}\left(\vec{y}_{v}\left(t_{2}-1\right)\right) \quad \forall t_{2}=1,2, \ldots, T_{2} .
$$

3) Source Mappings: With the fixed relaying operations for the relaying nodes, as defined above, an end-to-end deterministic channel results between the source and the destination nodes. Note that the input alphabet set at the source node is given by the source codebook of the unicast network $\mathcal{C}_{S}$. The deterministic $\mathrm{BC}$ is time invariant since the same relay mappings are used for all $t_{2}$ and is characterized by the functions

$$
\vec{y}_{D_{i}}=\psi_{i}\left\{\vec{x}_{S}\right\} \quad \forall i=1,2, \ldots, J .
$$

The capacity of the deterministic BC is well known (see [6] and [9]). In particular, the coding scheme described for the deterministic BC in [7], commonly referred to as the "Marton code," can be used and is described below succinctly.

A brief description of the Marton code is given here for completeness, we refer the reader to [7] for further details. The random code ensemble is constructed as follows. Consider a uniform distribution over $\mathcal{C}_{S}$, which is a collection of $\vec{X}_{S}$. The channel and the relay mapping $\psi_{i}$ induce the joint distribution 
over the random variables $\left(\vec{X}_{S}, \vec{Y}_{\mathcal{V}}\right)$. Create auxiliary random variables $\vec{U}_{D_{i}}$ such that $p_{\vec{X}, \vec{U}_{D_{1}}, \vec{U}_{D_{2}}, \ldots, \vec{U}_{D_{J}}}$ is the same as $p_{\vec{X}, \vec{Y}_{D_{1}}, \vec{Y}_{D_{2}}, \ldots, \vec{Y}_{D_{J}}} \cdot$

The set $\mathcal{T}_{\delta}^{T_{2}}\left(\vec{U}_{D_{i}}\right)$ of all typical $\vec{u}_{D_{i}}^{T_{2}}$ are binned into $2^{T_{1} T_{2} R_{i}}$ bins, where each bin index corresponds to a message, for $i=1,2, \ldots, J$. For each vector $\left(\vec{u}_{D_{1}}^{T_{2}}, \ldots, \vec{u}_{D_{J}}^{T_{2}}\right) \in \mathcal{T}_{\delta}^{T_{2}}\left(\vec{Y}_{D_{1}}, \ldots, \vec{Y}_{D_{J}}\right)$, there exists a sequence $\vec{x}_{S}^{T_{2}}\left(\vec{u}_{D_{1}}^{T_{2}}, \ldots, \vec{u}_{D_{J}}^{T_{2}}\right)$, since the channel is deterministic, such that $\left(\vec{x}_{S}^{T_{2}}, \vec{u}_{D_{1}}^{T_{2}}, \ldots, \vec{u}_{D_{J}}^{T_{2}}\right) \in \mathcal{T}_{\delta}^{T_{2}}\left(\vec{X}_{S}, \vec{Y}_{D_{1}}, \ldots, \vec{Y}_{D_{J}}\right)$.

4) Encoding: To transmit the message $\left(W_{1}, \ldots, W_{J}\right)$, the source tries to find a vector $\left(\vec{u}_{1}^{T_{2}}, \ldots, \vec{u}_{J}^{T_{2}}\right) \in$ $\mathcal{T}_{\delta}^{T_{2}}\left(\vec{U}_{1}, \ldots, \vec{U}_{J}\right)$ such that $\vec{u}_{i}^{T_{2}}$ is also in the bin with index $W_{i}$. If the source can find such a vector, it transmits $\vec{x}_{s}^{T_{2}}\left(\vec{u}_{1}^{T_{2}}, \ldots, \vec{u}_{J}^{T_{2}}\right)$. If the source cannot find such a sequence, it transmits a random sequence.

5) Decoding: The destination $D_{i}$ finds the bin in which the received vector $\vec{y}_{D_{i}}^{T_{2}}$ falls and decodes that bin index as the transmitted message.

\section{Performance Analysis}

First, the rate constraints for the Marton code are identified under which arbitrarily low probability of error is guaranteed provided a large enough $T_{2}$ is chosen. It is shown in [7] that this is guaranteed, provided the rate tuple $\left(R_{1}, \ldots, R_{J}\right)$ satisfies

$$
\sum_{j \in \mathcal{J}} R_{j}<\frac{1}{T_{1}} H\left(\vec{Y}_{D_{\mathcal{J}}}\right) \quad \forall \mathcal{J} \subseteq\{1, \ldots, J\}
$$

where $D_{\mathcal{J}}=\left\{D_{j}\right\}_{j \in \mathcal{J}}$.

Next, $H\left(\left\{\vec{Y}_{D_{i}}\right\}_{D_{i} \in L}\right)$ is evaluated with the relaying operations that was chosen using the following lemma.

Lemma 2: Given arbitrary $\epsilon>0, \exists T_{1}$ s.t.,

$$
H\left(\vec{Y}_{D_{\mathcal{J}}}\right) \geq T_{1}\left(\sum_{j \in \mathcal{J}} r_{j}-\epsilon\right) \quad \forall \mathcal{J} \subseteq\{1, \ldots, J\} .
$$

Proof: See Appendix B for the proof.

Using (11) and Lemma 2, it can be concluded that the rate tuple $\left(r_{1}, \ldots, r_{J}\right)$ is achievable.

Since $\left(r_{1}, \ldots, r_{J}\right)$ was chosen to be any point in $\bar{C}\left(Q_{p}\right)$, the region $\bar{C}\left(Q_{p}\right)$ is achievable. This proves the theorem.

\section{Gaussian Broadcast Networks (ProOF OF THEOREM 1)}

In this section, we prove the main claim of the paper, that for Gaussian broadcast networks, the cut-set bound is achievable to within a constant number of bits.

As for the deterministic network, only a layered network is considered here. A nonlayered network is handled in the same way as done in [1]. For the Gaussian network, while it is possible to do the inner code as done in the deterministic network and induce an end-to-end $\mathrm{BC}$; the induced $\mathrm{BC}$ would be a vector nonlinear non-Gaussian $\mathrm{BC}$ due to the complicated nature of the relay mappings. For general BCs, it is unknown whether a Marton scheme achieves rates within a constant gap of the cut-set bound.
Therefore, a different approach along the lines of [2], with the DSN approximation for the Gaussian network as a digital interface is used. The DSN is a deterministic network. The germane code for this deterministic network is constructed and then appropriately "lifted" to construct the code for the Gaussian network.

\section{A. Discrete Superposition Network (DSN)}

In [2], the recipe to construct a DSN corresponding to a Gaussian network is given. We start with the given Gaussian network defined by the channel model

$$
y_{\ell}[t]=\sum_{k \neq \ell} h_{k \ell}[t] x_{k}[t]+z_{\ell}[t] \quad \forall \ell \in \mathcal{V}
$$

where each node has unit power constraint and the noise has unit variance. We construct a corresponding DSN by defining the equivalent channel model in the DSN as follows:

$$
\hat{y}_{\ell}[t]=\left\lfloor\sum_{k \neq \ell}\left\lfloor h_{k \ell}[t]\right\rfloor \hat{x}_{k}[t]\right\rfloor
$$

where $\lfloor\ldots\rfloor$ lies in $\mathbb{Z}+\imath \mathbb{Z}$ and corresponds to quantizing the real and imaginary parts of the complex number by neglecting the fractional part. Further, the transmit alphabet in the DSN is restricted to a finite set, such that both the real and imaginary parts belong to the finite set with equally spaced points given by

$$
\frac{1}{\sqrt{2}}\left\{0,2^{-n}, \ldots, 1-2^{-n}\right\}
$$

where $n=\max _{(i, j) \in E}\left\lfloor\log \left|h_{i j}\right|\right\rfloor$. We will use $\hat{\mathcal{X}}, \hat{\mathcal{Y}}$ for the output and input alphabets in the DSN. Observe that we have used the caret sign to distinguish corresponding quantities for the DSN from the Gaussian network. This DSN model is also similar to the truncated deterministic model in [1], where the channel inputs and channel gains are complex.

\section{B. Unicast Network: Connection Between Gaussian and DSN}

First, the connection between Gaussian and DSN unicast networks, which was established in [2] is revisited.

The following lemma establishes a crucial relationship between the two networks by relating the cut-set bounds in the two networks

Lemma 3 (see [2, Th. 3.2]): There exists a constant $k_{1}=O(|\mathcal{V}| \log |\mathcal{V}|)$, such that if $R$ is the min-cut of a Gaussian unicast network, then $R-k_{1}$ is the min-cut for the corresponding DSN unicast network evaluated under product form distributions.

In [2], a coding scheme for the Gaussian network was presented, which used the corresponding DSN as a digital interface. A coding scheme for the DSN was first constructed and the coding scheme for the Gaussian network was constructed by defining an emulation function that operated on top of the DSN scheme. This strategy is revisited next.

1) Emulation Scheme for Relay Network: Consider a unicast Gaussian network and it's corresponding DSN unicast network. 
The transmitted and received symbols at node $v$ in the DSN is denoted by $\hat{x}_{v}$ and $\hat{y}_{v}$ and the transmitted and received symbols at node $v$ in the Gaussian network is denoted by $x_{v}$ and $y_{v}$, respectively. Let $\hat{\mathcal{X}}_{v}, \hat{\mathcal{Y}}_{v}$ be the output and input alphabets at node $v$ of the DSN and $\mathcal{X}_{v}, \mathcal{Y}_{v}$ the output and input alphabets for the Gaussian network.

The coding scheme for the DSN $n \|$ is comprised of the following:

1) A source codebook $f_{S}:\left[2^{T R}\right] \rightarrow \hat{\mathcal{X}}_{S}^{T}$, i.e., $\hat{x}_{S}^{T}=f_{S}(W)$.

2) The relay mappings $f_{v}: \hat{\mathcal{Y}}_{v}^{T} \rightarrow \hat{\mathcal{X}}_{v}^{T}$,i.e., $\hat{x}_{v}^{T}=f_{v}\left(\hat{y}_{v}^{T}\right)$ and

3) The destination decoder $g_{D}: \hat{\mathcal{Y}}_{D}^{T} \rightarrow\left[2^{T R}\right]$, i.e., $\hat{W}=$ $g_{D}\left(\hat{y}_{D}^{T}\right)$.

For the Gaussian network, the DSN coding scheme can be emulated on the Gaussian network using "emulation mappings" $e_{v}$ that convert the received vector in the Gaussian network to the received vector in the DSN given by

$$
e_{v}: \mathcal{Y}_{v}^{T} \rightarrow \hat{\mathcal{Y}}_{v}^{T}
$$

The emulation mapping along with the coding scheme of the DSN comprises the coding scheme for the Gaussian network.

The probability of error for emulation is defined as the probability that the emulated vector is different from the vector in the DSN and is given by

$$
\mathbb{P}\left\{\exists v: e_{v}\left(y_{v}^{T}(W)\right) \neq \hat{y}_{v}^{T}(W)\right\} .
$$

In [2], it has been shown that there exits an emulation mapping such that the probability of error for emulation can be made arbitrarily small for rate within a constant of the cut-set bound. This is stated more precisely in the following lemma.

Lemma 4 [2]: Given a zero-error coding scheme for the DSN unicast network of rate $R$, a pruned coding scheme of rate $R-\kappa$ (with $\kappa=\log (6|\mathcal{V}|-1)+11$ ) can be created for the DSN unicast network and an emulation scheme can be created for the Gaussian network with probability of emulation error lesser than $\epsilon$, for any arbitrary $\epsilon>0$.

Proof: For proof, refer [2, Th. 3.4].

\section{Coding Scheme for the Gaussian Broadcast Network}

Let us consider a specific rate vector $\left(r_{1}, \ldots, r_{J}\right)$ in the interior of $\bar{C}^{\mathrm{g}}$, the cut-set region for the Gaussian network. A coding scheme with rate vector a constant away from the rate vector $\left(r_{1}, \ldots, r_{J}\right)$ is constructed as follows.

1) Consider the corresponding DSN network to the Gaussian network. Next, construct the unicast network by adding a super-destination $D$ to both the Gaussian and the DSN network. This unicast network is further augmented by adding incoming edges from each of the destinations $D_{i}$ by a rate-limited wired link of capacity $r_{i}$.

The cut-set bound of the Gaussian unicast network is equal to $\sum_{i} r_{i}=: r$. The cut-set bound of the DSN unicast network (under product form distributions) is given by $\tilde{r} \geq r-k_{1}$ where $k_{1}=O(|\mathcal{V}| \log (|\mathcal{V}|))$ by Lemma 3. Theorem 2 then implies that there exists a zero-error coding scheme for the DSN unicast network at rate $r-k_{1}$.

2) Construct a $\left(2^{(r-\kappa) T_{1}}, T_{1}\right)$-pruned coding scheme for this DSN unicast network at rate $r-k$, with $k=k_{1}+\kappa=$
$O(|\mathcal{V}| \log (|\mathcal{V}|))$ and $\kappa=\log (6|\mathcal{V}|-1)+11$, as given by Lemma 4. This scheme can be emulated on the Gaussian unicast network with an arbitrarily small error probability.

3) The relay mappings from the DSN unicast network can then be used to create a coding scheme for the DSN broadcast network as described in Section III-B3. This is done using the relay mapping to construct a deterministic end-to-end $\mathrm{BC}$ and then using the Marton code.

Recall that the coding scheme is over $T_{1} T_{2}$ time instants, where each set of $T_{1}$ time instants is treated as a block and the vector $\vec{x}\left(t_{2}\right)$ denotes $x$ over the $T_{1}$ time instants corresponding to the $t_{2}$ th block and is denoted by $\vec{x}\left(t_{2}\right)$. The relay mappings are given by $\hat{f}_{v}$ and operates over the blocks of $T_{1}$ time instants.

4) For the Gaussian broadcast network, the emulation mapping is then used to emulate the received vectors on the DSN and hence convert the scheme for the DSN broadcast network to a scheme for the Gaussian broadcast network.

\section{Performance Analysis}

First, the rates that can be achieved for the DSN broadcast network is characterized. As seen in Section III-C, this is given by

$$
\sum_{j \in \mathcal{J}} R_{j}<\frac{1}{T_{1}} H\left(\vec{Y}_{D_{\mathcal{J}}}\right) \quad \forall \mathcal{J} \subseteq\{1, \ldots, J\} .
$$

Note that $\vec{Y}_{D_{\mathcal{J}}}$ is obtained by assuming a uniform distribution over the pruned codebook for the DSN unicast network. The following lemma analogous to Lemma 2 characterizes $H\left(\vec{Y}_{D_{\mathcal{J}}}\right)$.

Lemma 5: Given arbitrary $\epsilon>0, \exists T_{1}$ s.t.,

$$
H\left(\vec{Y}_{D_{\mathcal{J}}}\right) \geq T_{1}\left(\sum_{j \in \mathcal{J}} r_{j}-k-\epsilon\right) \quad \forall \mathcal{J} \subseteq\{1, \ldots, J\} .
$$

Proof: The proof of this lemma is the same as the proof of Lemma 2 with $r-k$ replacing $r$ as the rate of the DSN unicast scheme.

Therefore, the rates $R_{j}=r_{j}-k$ can be achieved for the DSN broadcast network.

Lemma 4 ensures an emulation mapping with arbitrarily small emulation error probability, and thus, the rate vector $\left(r_{1}-k, \ldots, r_{J}-k\right)$ can be achieved for the Gaussian broadcast network. This completes the proof of Theorem 1 .

\section{Generalizations}

In this section, we present various generalizations of our result, for half-duplex networks in Section V-A, for networks with multiple antenna in Section V-B and for broadcast wireless networks, where some set of nodes demand the same information and other nodes demand independent information in Section V-C.

\section{A. Half-Duplex Networks}

Our discussion so far has been restricted to the context of full duplex scenario. A network is said to be half-duplex if the nodes in the network can either transmit or receive information, but not do both simultaneously. Therefore, the network needs to be 
scheduled by specifying which nodes are listening and which nodes are transmitting at any given time instant. Let the set of all possible half-duplex schedules at any time instant be $\mathcal{H}$. An edge $e_{i j}$ is said to be active at time slot $t$ if $v_{i}$ is transmitting and $v_{j}$ is receiving at that time slot.

Consider $T$ time slots and at any time instant $t$, let $h_{t} \in \mathcal{H}$ be the half-duplex schedule used, and $h^{T}$ be the sequence $h_{1}, h_{2}, \ldots, h_{T}$. We consider only static schedules here, that is, schedules that are specified a priori and do not vary depending on dynamic parameters like channel noise. For any static schedule $h^{T}$, we can unfold the network graph with respect to that schedule. This unfolding procedure is the same as performed in [1] by: $v_{1}[t]$ is connected to $v_{2}[t+1]$ with a link $h_{v_{1}, v_{2}}$ only when $e_{v_{1} v_{2}}$ is active at time slot $t$.

Given that the network is operated under a schedule $h^{T}$, we define the set of all rate pairs achievable as the capacity region under the schedule $h^{T}$. An outer bound on the capacity region under the schedule $h^{T}$ is given by the cut-set bound in the unfolded layered network corresponding to the schedule. This rate can be achieved within a constant gap by using Theorem 1 . Thus, for any schedule $h^{T}$, any rate tuple within the constant $k=O(|\mathcal{V}| \log (|\mathcal{V}|))$ of the cut-set bound can be achieved (to within a constant number of bits) using that schedule and then using the scheme of Theorem 1 for the unfolded layered network. Now, we can optimize over all schedules $h^{T} \in \mathcal{H}$ allowed under the half-duplex constraints. Thus, the capacity region of the network under static half-duplex scheduling is the union over all possible schedules of the capacity region under schedule $h^{T}$.

\section{B. Multiple Antenna Networks}

In this section, we consider the implication of having multiple antenna elements at each of the nodes in the network. Suppose $v$ possesses $m_{v}$ antenna elements, which are used for both transmission and reception. The basic result for multiantenna broadcast networks is the following.

Theorem 3: For the Gaussian multiantenna broadcast network, given a tuple $\left(r_{1}, \ldots, r_{J}\right) \in \overline{\mathcal{C}}$ that lies in the cut-set region, any rate tuple $\left(R_{1}, \ldots, R_{J}\right)$ satisfying

$$
\sum_{j \in \mathcal{J}} R_{j} \leq \sum_{j \in \mathcal{J}} r_{j}-k \quad \forall \mathcal{J} \subseteq(1,2, \ldots, J)
$$

is achievable where $k=O(M \log M)$ with $M=\sum_{v \in \mathcal{V}} m_{v}$ being a constant which depends only on the number of nodes and the number of antennas in each node, and not on the channel coefficients or the power constraints.

Proof: The proof is essentially the same as the one for the single antenna case in Section IV. We can essentially think of each antenna as a distinct node with infinite capacity edges between the various antennas of a given node and then the proof for the single antenna case just goes through.

\section{Broadcast-Cum-Multicast}

The broadcast network comprised of a single source $S$ and destinations $D_{1}, D_{2}, \ldots, D_{J}$ demanding independent messages at rates $R_{1}, R_{2}, \ldots, R_{J}$. Suppose that in addition there are also other multicast destinations $M_{1}, M_{2}, \ldots, M_{L}$ that demand all the messages transmitted by the source. We call such a network a broadcast-cum-multicast network. In this section, we will show that even for such networks, the cut-set bound is achievable to within a constant number of bits. This network is a generalization of both the multicast network considered in [1] and the broadcast network considered in the previous sections. The broadcast-cum-multicast traffic model is also considered in [18] and [19] in the context of wireline and linear-deterministic networks, respectively, where it is also noted that the source should carefully choose the coding coefficients such that the destinations can decode, resonating with the "receiver-centric" viewpoint suggested here.

First, we note that the cut-set bound for the broadcast-cummulticast network is given by the cut-set bound for the broadcast network, along with the cut-set constraints for each multicast receiver. In particular for the Gaussian broadcast-cum-multicast network, if $\left(R_{1}, \ldots, R_{J}\right)$ is achievable, then there exists a joint distribution $Q$ such that

$$
R_{\mathcal{J}} \leq \min _{\Omega \in \Lambda_{\mathcal{J}}} I\left(X_{\Omega} ; Y_{\Omega^{c}} \mid X_{\Omega^{c}}\right) \quad \forall \mathcal{J} \subseteq[J]
$$

and in addition, the sum rate is constrained by each of the multicast destinations (since all these destinations demand all the messages transmitted by the source)

$$
R_{[J]} \leq \min _{i \in[L]} \min _{\Omega \in \Lambda_{M_{i}}} I\left(X_{\Omega} ; Y_{\Omega^{c}} \mid X_{\Omega^{c}}\right)
$$

The set of all rate tuples inside the cut-set bound is then denoted by $\bar{C}$.

The main result for the wireless broadcast-cum-multicast network is that any rate a constant away from cut-set bound is achievable.

Theorem 4: For the Gaussian broadcast-cum-multicast network, given a tuple $\left(r_{1}, \ldots, r_{J}\right) \in \overline{\mathcal{C}}$ that lies in the cut-set region, any rate tuple $\left(R_{1}, \ldots, R_{J}\right)$ satisfying

$$
\sum_{j \in \mathcal{J}} R_{j} \leq \sum_{j \in \mathcal{J}} r_{j}-k \quad \forall \mathcal{J} \subseteq(1,2, \ldots, J)
$$

is achievable where $k=O(|\mathcal{V}| \log |\mathcal{V}|)$ is a constant which depends only on the number of nodes, and not on the channel coefficients or the power constraints

To prove this result, we follow an approach similar to the one we took for broadcast networks. First, we will prove a result for deterministic broadcast-cum-multicast networks. Second, we show that the Gaussian network can emulate the deterministic superposition network with a constant rate loss. These two steps are completed in the rest of this section.

1) Deterministic Broadcast-Cum-Multicast Network: The next lemma shows that for the deterministic broadcast-cum-multicast network, the cut-set bound evaluated under product form distributions is achievable.

Lemma 6: For the deterministic broadcast-cum-multicast network, the cut-set bound under product-form distributions is 
achievable, i.e., a rate vector $\left(R_{1}, \ldots, R_{K}\right)$ is achievable if for every $\mathcal{J} \in[J]$ there is some product probability distribution $Q_{p}$, such that

$$
\begin{aligned}
R_{\mathcal{J}} & \leq \min _{\Omega \in \Lambda_{\mathcal{J}}} I\left(X_{\Omega} ; Y_{\Omega^{c}} \mid X_{\Omega^{c}}\right) \\
& =\min _{\Omega \in \Lambda_{\mathcal{J}}} H\left(Y_{\Omega^{c}} \mid X_{\Omega^{c}}\right) \quad \forall \mathcal{J} \subseteq[J] \text { and } \\
R_{[J]} & \leq \min _{i \in[L]} \min _{\Omega \in \Lambda_{M_{i}}} I\left(X_{\Omega} ; Y_{\Omega^{c}} \mid X_{\Omega^{c}}\right) \\
& =\min _{i \in[L]} \min _{\Omega \in \Lambda_{M_{i}}} H\left(Y_{\Omega^{c}} \mid X_{\Omega^{c}}\right) .
\end{aligned}
$$

Proof: The coding scheme proceeds exactly in the same way as in the proof of Theorem 2, elaborated in Section III-B. Here, we sketch the proof by highlighting the differences for the broadcast-cum-multicast network.

1) The broadcast-cum-multicast network is converted into a multicast network by adding a super-destination $M_{0}$ which has links from each of the destinations $D_{i}$ by a rate-limited link of capacity $r_{i}$. There are multicast destinations $M_{0}, M_{1}, \ldots, M_{L}$. If $\left(r_{1}, \ldots, r_{J}\right)$ is in the cut-set region of the broadcast-cum-multicast network (evaluated under product distributions), then the min-cut of the multicast network (evaluated under product distributions) is equal to $\sum_{j} r_{j}$.

2) For the multicast network, a zero-error coding scheme is employed that operates over $T_{1}$ time instants, which achieves the rate $\sum_{j} r_{j}$.

3) The multicasting scheme creates an end-to-end deterministic broadcast-cum-multicast channel between the source $S$ and the destinations $D_{1}, \ldots, D_{J}, M_{1}, \ldots, M_{L}$ over vectors of $T_{1}$ symbols.

4) Consider the induced deterministic broadcast-cum-multicast channel. We use a Marton code for destinations $D_{1}, \ldots, D_{J}$ of block length $T_{2}$ using the auxiliary random variables $\vec{U}_{i}=\vec{Y}_{D_{i}}$. The auxiliary random variable $\vec{U}_{i}^{T_{2}}$ is binned into $2^{T_{1} T_{2} R_{i}}$ bins corresponding to the different values of the message $W_{i}$. The source takes the messages $\left(W_{1}, \ldots, W_{J}\right)$ and finds a jointly typical $\left(\vec{U}_{1}, \ldots, \vec{U}_{J}\right)$. This implies that there exists a $\vec{X}^{T_{2}}$ such that $\left(\vec{X}, \vec{U}_{1}, \ldots, \vec{U}_{J}\right)$ is jointly typical. This $\vec{X}^{T_{2}}$ is transmitted. The broadcast destination $D_{i}$ finds the bin in which $\vec{Y}_{D_{i}}^{T_{2}}$ falls and declares that as the message. The multicast destinations find the $\vec{X}^{T_{2}}$ which is jointly typical with the received $\vec{Y}^{T_{2}}$ and declares the corresponding $\left(W_{1}, \ldots, W_{J}\right)$ as the message. This coding scheme achieves low probability of error as long as

$$
\begin{aligned}
R_{\mathcal{J}} & \leq \frac{1}{T_{1}} H\left(\vec{Y}_{D_{\mathcal{J}}}\right) \quad \forall \mathcal{J} \subseteq[J] \\
R_{[J]} & \leq \frac{1}{T_{1}} H\left(\vec{Y}_{M_{i}}\right) \quad \forall i \in[L] .
\end{aligned}
$$

5) Since the multicasting scheme achieves rate $\sum_{i} r_{i}$, by performing a calculation similar to Lemma 2 , we can assert that

$$
\begin{gathered}
H\left(\vec{Y}_{D_{\mathcal{J}}}\right) \geq T_{1} \sum_{j \in \mathcal{J}} r_{j} \quad \forall \mathcal{J} \subseteq[J] \\
H\left(\vec{Y}_{M_{i}}\right) \geq T_{1} \sum_{i} r_{i} \quad \forall i \in[L] .
\end{gathered}
$$

This implies that $\left(R_{1}, \ldots, R_{J}\right)=\left(r_{1}, \ldots, r_{J}\right)$ is feasible, for any $\left(r_{1}, \ldots, r_{J}\right)$ in the cut-set region of the broadcast-cum-multicast network (evaluated under product distributions).

Corollary 3: For the linear-deterministic broadcast-cummulticast network, the cut-set bound is achieved. For the deterministic broadcast-cum-multicast channel (a deterministic broadcast-cum-multicast network in the absence of relays and destination cooperation), the cut-set bound is achieved.

Proof: In the latter case, the cut-set bound under product form distribution is the same as the cut-set bound under general distributions since there is only one transmitting node in the network. The former case can be proved by showing that the cut-set bound for linear-deterministic networks is optimized by product form distributions.

2) Gaussian Broadcast-Cum-Multicast Network: The proof for the Gaussian broadcast-cum-multicast network is the same as the proof for the Gaussian broadcast network, except that instead of starting with an emulation scheme for the Gaussian relay network, we start with an emulation scheme for the Gaussian multicast network by a DSN multicast network, which is available in [2, Th. 5.3]. Then, the coding scheme of Lemma 6 for the DSN broadcast-cum-multicast network can be emulated on the Gaussian broadcast-cum-multicast network. The proof then follows a similar path as the proof of Theorem 1, as elaborated in Section IV-C and is omitted here to avoid repetition.

\section{DISCUSSION}

In this section, we will show an approximate reciprocity between Gaussian broadcast networks and Gaussian multi-source networks. Then, we mention the CSI requirements for the presented scheme for Gaussian broadcast networks and contrast it with the CSI requirements for the Gaussian multi-source networks. We finish the discussion section with some comments on the capacity gap.

\section{A. Approximate Reciprocity in Gaussian Multi-Source and Broadcast Networks}

In this section, we will demonstrate that there is an approximate reciprocity in the capacity regions of a Gaussian multi-source network and the corresponding reciprocal Gaussian broadcast network.

In our model, we have assumed, without loss of generality, the average transmit power constraint of unity at each node. We have also assumed that the reciprocal network, in addition to having the same channel coefficients, also has unit power constraints at each node. However, it is not clear if this is the "right" way of defining the corresponding reciprocal network. For instance, in [22] and [23], MAC-BC duality was shown under the assumption of same total transmit power in both networks; however, this power could be divided amongst the nodes in a different manner in the forward and reciprocal networks. Under this assumption, it was shown that the capacity region of the two networks was identical. However, since we are concerned only 
about approximate reciprocity in this section, which is a weaker form of reciprocity, our definition of unit power constraint everywhere will be sufficient.

In [5] and [17], a coding scheme is given for the Gaussian network with many sources and is shown to achieve the cut-set bound region within a constant gap, which depends only on the network gain. In Section IV, we have showed that for the Gaussian broadcast network also, we can achieve the cut-set bound region within a constant gap. As a result, to show that the capacity region of the two networks are themselves within a constant gap, which depends only on the network topology and not on the channel gains, all we need to do is to observe that cut-sets of the reciprocal networks are within a constant gap of each other. Note that the cut-set bound corresponds to MIMO point-to-point channel where all the nodes on the source side of the nodes can be thought of as transmit antennas and all the nodes on the destination side can be thought of as receive antennas. The relationship then between a cut in a network and the corresponding cut in the reciprocal network is the same as the relationship between a MIMO channel with channel matrix $H$ and the reciprocal MIMO channel with the channel matrix $H^{T}$. The reciprocity of MIMO channel has been shown in [25], under equal total transmit power, i.e., the capacity of the two networks is the same. It can be shown (see proof of Lemma 6.6 in [1], for instance) that restricting to per node power constraint only leads to a loss which does not depend on the channel gains. Therefore, we can show that the cut-set bounds are approximately reciprocal, which implies that the capacity regions are approximately reciprocal as well.

\section{B. Channel State Information (CSI)}

We now examine the CSI required at the various nodes for the schemes proposed in Sections III and IV for deterministic and Gaussian broadcast networks.

1) Deterministic Broadcast Networks: While we have used an arbitrary coding scheme for the DSN relay network in constructing the coding scheme for the Gaussian network, we could as a special instance choose to work with the random coding scheme. Once we use a random coding scheme for the DSN relay network, the relays do only random coding and do not, therefore, need to know the channel state.

Thus, for deterministic networks, the following CSI is required.

1) All non-source non-destination nodes are unaware of any CSI.

2) We assume that each destination knows the distribution of the received vector $\vec{Y}_{D_{i}}$ and bins the set of all typical vectors into $2^{T R_{i}}$ bins corresponding to the messages, and uses this as the decoding rule.

3) The transmitter is assumed to have full CSI, and knows the relay mappings at all nodes and also the binning scheme at the destinations. The transmitter constructs the codebook using the same binning scheme as the receiver.

Thus, this scheme has the interesting property that if the transmitter had all knowledge, the intermediate nodes have zero knowledge and the destination has a little knowledge (about the distribution of the received vector), then the same rate can be achieved as the complete CSI case.
This is dual to the situation of the multi-source single-destination network, where the receiver having full knowledge, intermediate nodes having zero knowledge and the transmitters having a little knowledge (about the distribution of the transmitted vector) can achieve the same rate as full channel knowledge.

2) Gaussian Broadcast Network: For Gaussian networks, the following CSI is required:

1) All non-source non-destination nodes need to know enough CSI to emulate the corresponding nodes in the DSN. This implies that each node $v$ needs to use the received vector distribution to pick a pruned subset of the typically received vectors in the corresponding DSN in the emulation scheme. This information is contained in the CSI of the local neighborhood of a node (channel coefficients of all edges connected to a given node).

2) We assume that each destination knows the distribution of the received vector in the corresponding DSN $\vec{Y}_{D_{i}}$, and the transmitted rate $R_{i}$. The destination maps bins the set of all typical vectors into $2^{T_{1} T_{2} R_{i}}$ bins corresponding to the messages, and uses this as the decoding rule.

3) The transmitter is assumed to have full CSI, and knows the mappings used at all the nodes and also the binning scheme at the destinations. The transmitter then uses the same binning scheme used at the receiver.

This scheme has the interesting property that if the transmitter had all knowledge, the intermediate nodes and the destination have local knowledge, then the same rate can be achieved as the complete CSI case.

\section{Capacity Gap}

The gap between the sum rate of the proposed achievable scheme and the outer bound for the Gaussian broadcast network is given by $O(|\mathcal{V}| \log |\mathcal{V}|)$. This gap is independent of the power constraints and the channel coefficients. This is the same as the gap obtained for relay networks in [2], since we are using the same emulation scheme to connect the Gaussian and DSN networks in our scheme. The results in [1] and [5] give better approximation guarantees for Gaussian relay networks with the constant gap being linear in the number of nodes: $O(|\mathcal{V}|)$. These results are obtained directly without the use of deterministic superposition network. It may be possible to obtain $O(|\mathcal{V}|)$ gaps for the broadcast scenario either by completely bypassing the deterministic models and working directly with the Gaussian network or by using the truncated deterministic model of [1] which is a better approximation of Gaussian network than the DSN model of [2]. This is one possible direction for future work.

\section{APPENDIX A \\ PROOF OF LEMMA 1}

Consider any cut $\Omega$ such that $S \in \Omega$ and $D \in \Omega^{c}$. There are two components that contribute to the value of the cut: one part $c_{1}$ comes from the wired links and the other part $c_{2}$ that comes from the original network. Let $\mathcal{J}$ be such that $D_{\mathcal{J}^{c}} \subseteq \Omega$ and $D_{\mathcal{J}} \subseteq \Omega^{c}$, this implies that the wired links of capacity $c_{1}=\sum_{j \in \mathcal{J}^{c}} r_{j}$ are included in the cuts. Recall $\bar{C}_{\mathcal{J}}(Q)$ denotes the value of the cut-set bound evaluated under the distribution 
$Q$ for separating the source from the set $D_{\mathcal{J}}$. As the cut $\Omega$ separates $S$ from $D_{\mathcal{J}}$ the value of cut gained from the original network is bigger than $\bar{C}_{\mathcal{J}}(Q)$. Furthermore, since $\left(r_{1}, \ldots, r_{J}\right) \in$ $\bar{C}(Q), \sum_{j \in \mathcal{J}} r_{j} \leq \bar{C}_{\mathcal{J}}(Q)$. This implies that the value of cut gained from the original network is bigger than this value: $c_{2} \geq \sum_{j \in \mathcal{J}} r_{j}$. Thus, the total value of the cut is $c=c_{1}+c_{2} \geq$ $\sum_{j} r_{j}$. The min-cut value is actually equal to $\sum_{j} r_{j}$ since the cut that separates $D_{1}, \ldots, D_{J}$ from $D$ has value $\sum_{j} r_{j}$.

\section{APPENDIX B \\ PROOF OF LEMMA 2}

Let the min-cut between the source to the destination be $r$. Since the relaying scheme can achieve any rate close to the cut-set bound for large enough $T_{1}$, the information transmitted by all the sinks to the super-sink $\mathrm{D}$ should be greater than rate $r$, therefore for any subset $\mathcal{J} \subseteq\{1,2, \ldots, J\}$

$$
\begin{aligned}
T_{1} r & \leq H\left(\vec{X}_{D_{1}}, \ldots, \vec{X}_{D_{\mathcal{J}}}\right) \\
& \leq H\left(\vec{X}_{D_{\mathcal{J}}}\right)+H\left(\vec{X}_{D_{\mathcal{J}^{c}}}\right) \\
& \leq H\left(\vec{X}_{D_{\mathcal{J}}}\right)+\sum_{j \in \mathcal{J}^{c}} H\left(\vec{X}_{D_{j}}\right) \\
& =H\left(\vec{X}_{D_{\mathcal{J}}}\right)+\sum_{j \in \mathcal{J}^{c}} H\left(X_{D_{j}}^{T_{1}}\right) \\
& =H\left(\vec{X}_{D_{\mathcal{J}}}\right)+\sum_{j \in \mathcal{J}^{c}} H\left(X_{D_{j}}(1), \ldots, X_{D_{j}}\left(T_{1}\right)\right) \\
& \leq H\left(\vec{Y}_{D_{\mathcal{J}}}\right)+\sum_{j \in \mathcal{J}^{c}} \sum_{t=1}^{T_{1}} H\left(X_{D_{j}}(t)\right) \\
& \leq H\left(\vec{Y}_{D_{\mathcal{J}}}\right)+\sum_{j \in \mathcal{J}^{c}} T_{1} r_{j}
\end{aligned}
$$

where the last inequality follows due to the rate constraint on the links. Thus, we get

$$
\frac{1}{T_{1}} H\left(\vec{Y}_{D_{\mathcal{J}}}\right) \geq r-\sum_{j \in \mathcal{J}^{c}} r_{j}
$$

Furthermore, the min-cut (under product distributions) is $r=$ $\sum_{i} r_{i}$ by Lemma 1 , and this gives

$$
\frac{1}{T_{1}} H\left(\vec{Y}_{D_{\mathcal{J}}}\right) \geq \sum_{j \in \mathcal{J}} r_{j} \quad \forall \mathcal{J} \subseteq\{1,2, \ldots, J\}
$$

\section{ACKNOWLEDGMENT}

The authors would like to thank the associate editor and the anonymous reviewers for providing detailed reviews which helped improve the presentation of the paper.

\section{REFERENCES}

[1] A. S. Avestimehr, S. N. Diggavi, and D. N. C. Tse, "Wireless network information flow: A deterministic approach," IEEE Trans. Inf. Theory, vol. 57, no. 4, pp. 1872-1905, Apr. 2011.

[2] M. Anand and P. R. Kumar, "A digital interface for Gaussian relay and interference networks: Lifting codes from the discrete superposition model," IEEE Trans. Inf. Theory, vol. 57, no. 5, pp. 2548-2564, May 2011.

[3] T. M. Cover and J. A. Thomas, Elements of Information Theory, ser. Wiley Series in Telecommunications and Signal Processing. New York: Wiley, 2006
[4] A. El Gamal, "On information flow in relay networks," in Proc. IEEE Nat. Telecommun. Conf., Nov. 1981, vol. 2, pp. D4.1.1-D4.1.4.

[5] S. H. Lim, Y.-. H. Kim, A. El Gamal, and S.-Y. Chung, "Noisy network coding," IEEE Trans. Inf. Theory, vol. 57, no. 5, pp. 3132-3152, May 2011.

[6] K. Marton, "A coding theorem for the discrete memoryless broadcast channel," IEEE Trans. Inf. Theory, vol. IT-25, no. 3, pp. 306-311, May 1979.

[7] A. El Gamal and E. Van der Meulen, "A proof of Marton's coding theorem for the discrete memoryless broadcast channel," IEEE Trans. Inf. Theory, vol. IT-27, no. 1, pp. 120-122, Jan. 1981.

[8] Y. Liang and V. V. Veeravalli, "Cooperative relay broadcast channels," IEEE Trans. Inf. Theory, vol. 53, no. 3, pp. 900-928, Mar. 2007.

[9] M. S. Pinsker, "Capacity of noiseless broadcast channels," Probl. Pered. Inf., vol. 14, no. 2, p. 2834, Apr.-Jun. 1978, (translated in Probl. Inf. Transm., pp. 97-102, Apr.-Jun. 1978).

[10] P. Bergmans, "Random coding theorem for broadcast channels with degraded components," IEEE Trans. Inf. Theory, vol. IT-19, no. 2, pp. 197-207, Mar. 1973.

[11] H. Weingarten, Y. Steinberg, and S. Shamai, "The capacity region of the Gaussian multiple-input multiple-output broadcast channel," IEEE Trans. Inf. Theory, vol. 52, no. 9, pp. 3936-3964, Sep. 2006.

[12] M. X. Goemans, S. Iwata, and R. Zenklusen, "An algebraic framework for wireless information flow," presented at the Allerton Conf Commun., Control, Comput., Sep. 2009.

[13] S. M. S. Yazdi and S. A. Savari, "A combinatorial study of linear deterministic relay networks," presented at the Allerton Conf. Commun., Control, Comput., Sep. 2009.

[14] A. Raja, V. Prabhakaran, and P. Viswanath, "Reciprocity in linear deterministic networks under linear coding," presented at the IEEE Inf Theory Workshop, Volos, Greece, Jun. 2009.

[15] S. Kannan, A. Raja, and P. Viswanath, "Approximately optimal broadcasting in wireless networks," presented at the IEEE Int. Conf. Signal Process. Commun., Bangalore, India, Jul. 2010.

[16] S. Kannan, A. Raja, and P. Viswanath, "Approximately optimal broadcasting-cum-multicasting in wireless networks," presented at the IEEE Int. Symp. Inf. Theory, St. Petersburg, Russia, Aug. 2011.

[17] E. Perron, "Information-theoretic secrecy for wireless networks," Ph.D. dissertation, École Polytechnique Fédéral de Lausanne, Lausaane, Switzerland, Sep. 2009.

[18] R. Koetter and M. Médard, "An algebraic approach to network coding," IEEE Trans. Netw., vol. 11, no. 5, pp. 782-795, Oct. 2003.

[19] M. Kim, E. Erez, E. M. Yeh, and M. Médard, Deterministic network model revisited: An algebraic network coding approach [Online]. Available: http://arxiv.org/abs/1103.0999

[20] R. Ahlswede, N. Cai, S.-Y. R. Li, and R. W. Yeung, "Network information flow," IEEE Trans. Inf. Theory., vol. 46, no. 4, pp. 1204-1216, Jul. 2000.

[21] S. Riis, "Reversible and irreversible information networks," IEEE Trans. Inf. Theory., vol. 53, no. 11, pp. 4339-4349, Nov. 2007.

[22] S. Vishwanath, N. Jindal, and A. J. Goldsmith, "Duality, achievable rates and sum-rate capacity of Gaussian MIMO broadcast channel," IEEE Trans. Inf. Theory., vol. 49, no. 10, pp. 2658-2668, Oct. 2003.

[23] P. Viswanath and D. Tse, "Sum capacity of the multiple antenna Gaussian broadcast channel and uplink-downlink duality," IEEE Trans. Inf. Theory., vol. 49, no. 8, pp. 1912-1923, Aug. 2003.

[24] M. M. Butt, G. Caire, and R. R. Müller, Linear finite-field deterministic networks with many sources and one destination [Online]. Available: http://arxiv.org/abs/1001.2464.

[25] E. Telatar, "Capacity of multi-antenna Gaussian channels," Eur. Trans. Telecomm., vol. 10, pp. 585-596, Nov. 1999

[26] D. Vasudevan and S. B. Korada, "Polymatroidal flows on two classes of information networks," IEEE Trans. Inf. Theory, vol. 57, no. 1, pp. 227-233, Jan. 2011.

[27] R. Dougherty, K. Zeger, and C. Freiling, "Nonreversibility and equivalent constructions of multiple-unicast networks," IEEE Trans. Inf. Theory, vol. 52, no. 11, pp. 5067-5077, Nov. 2006.

Sreeram Kannan received the B.E. degree in electronics and communication engineering from the College of Engineering, Guindy, Anna University, and the M.E. degree from the Indian Institute of Science, Bangalore, in 2008. Since 2008, he has been a Ph.D. student at the University of Illinois Urbana-Champaign. His research interests include information theory and wireless networks. Mr. Kannan is a recipient of the S.V.C. Aiya medal from the Indian Institute of Science (2008), a recipient of Qualcomm Roberto Padovani Scholarship (2010), a corecipient of Intel India Student Research Contest first prize (2006), and a corecipient of the Qualcomm Cognitive Radio Contest prize (2010). 
Adnan Raja received his B.Tech. in 2006 from the Department of Electrical Engineering at the Indian Institute of Technology, Bombay, and his M.S. and Ph.D. from the Department of Electrical and Computer Engineering at the University of Illinois at Urbana-Champaign in 2008 and 2012 respectively. He is currently a post-doctoral researcher at Stanford University. His research interests are in information theory and wireless communication.
Pramod Viswanath (S'98-M'03-SM'10) received the Ph.D. degree in electrical engineering and computer science from the University of California at Berkeley, Berkeley, in 2000. He was a member of technical staff at Flarion Technologies until August 2001 before joining the Electrical and Computer Engineering Department, University of Illinois at Urbana-Champaign (UIUC), Urbana. Dr. Viswanath is a recipient of the Xerox Award for Faculty Research from the College of Engineering at UIUC (2010), the Eliahu Jury Award from the Electrical Engineering and Computer Science Department of the University of California at Berkeley (2000), the Bernard Friedman Award from the Mathematics Department of the University of California at Berkeley (2000), and the National Science Foundation (NSF) CAREER Award (2003). He was an Associate Editor of the IEEE TRANSACTIONS ON INFORMATION THEORY for the period 2006-2008 